\title{
Unicircular structure of the Brassica hirta mitochondrial genome
}

\author{
Jeffrey D. Palmer and Laura A. Herbon ${ }^{1}$ \\ Department of Biology, University of Michigan, Ann Arbor, MI 48109, USA
}

Summary. Restriction mapping studies reveal that the mitochondrial genome of white mustard (Brassica hirta) exists in the form of a single circular $208 \mathrm{~kb}$ chromosome. The $B$. hirta genome has only one copy of the two sequences which, in several related Brassica species, are duplicated and undergo intramolecular recombination. This first report of a plant mitochondrial DNA that does not exist in a multipartite structure indicates that high frequency intramolecular recombination is not an obligatory feature of plant mitochondrial genomes. Heterologous filter hybridizatios reveal that the mitochondrial genomes of $B$. hirta and $B$. campestris have diverged radically in sequence arrangement, as the result of approximately 10 large inversions. At the same time, however, the two genomes are similar in size, sequence content, and primary sequence.

Key words: Restriction map - Circular chromosome Recombination repeat - Inversion

\section{Introduction}

Plant mitochondrial DNAs (mtDNAs) are biwilderingly large in size and complex in sequence organization. The smallest known plant mtDNAs, from species of Brassica Lebacq and Vedel 1981; Palmer et al. 1983a) and Oenothera (Brennicke 1980), are on the order of 200 $\mathrm{kb}$ in size, larger than any animal, fungal or protist mtDNAs (Sederoff 1984). Moreover, certain plant

1 Present address: Department of Landscape Architecture and Regional Planning, University of Massachusetts, Amherst, MA 01003, USA

Offprint requests to: J. D. Palmer
mtDNAs are well over $2,000 \mathrm{~kb}$ in size (Ward et al. 1981), yet there is no evidence that these plant genomes contain significantly more genes than non-plant mtDNAs.

All plant mtDNAs characterized thus far exhibit a complex multipartite organization, in which a circular master chromosome, carrying the entire sequence complexity of the genome, is resolved into smaller subgenomic circles via recombination at one or more pairs of repeated sequences. This peculiar structural organization was first shown for the tripartite $218 \mathrm{~kb}$ genome of Brassica campestris, which recombines at a $2 \mathrm{~kb}$ repeat element to form subgenomic circles of $135 \mathrm{~kb}$ and $83 \mathrm{~kb}$ (Palmer and Shields 1984). Tricircular mitochondrial genomes have also been documented in spinach, radish and three other Brassica species (Stern and Palmer 1986; Palmer and Herbon 1986 and unpublished data). Much more complex organizations, resulting from recombination at a larger number of duplication elements, have been reported for the cereals maize (Lonsdale et al. 1984) and wheat (Falconet et al. 1984; Quetier et al. 1985).

In this report, we show that high frequency intramolecular recombination and a multipartite organization are not obligatory features of all plant mitochondrial genomes. Instread, we show that the mitochondrial genome of $B$. hirta, unlike that of related Brassica species, exists as a single size class of $208 \mathrm{~kb}$ circles. Moreover, we find that the sequence organization of the $B$. hirta genome differs extensively from that of $B$. campestris, even though the two genomes are highly similar in size and primary sequence.

\section{Materials and methods}

Mitochondrial DNA was isolated from six week old green leaves of white mustard (Brassica hirta; syn. Sinapis alba), turnip (Bras- 


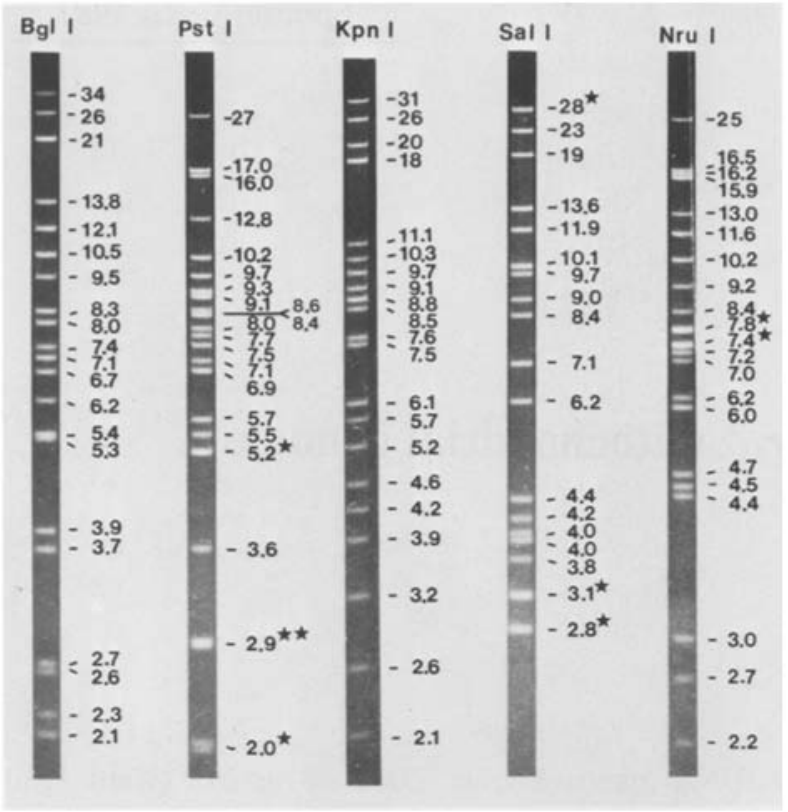

Fig. 1. Separation of $B$. hirta mtDNA restriction fragments on a $0.7 \%$ agarose gel. Fragment sizes are in $\mathrm{kb}$. Doublet intensity bands are marked with a star and triplet bands with two stars. Only fragments $2 \mathrm{~kb}$ and larger are shown; smaller fragments are indicated on the map shown in Fig. 4A

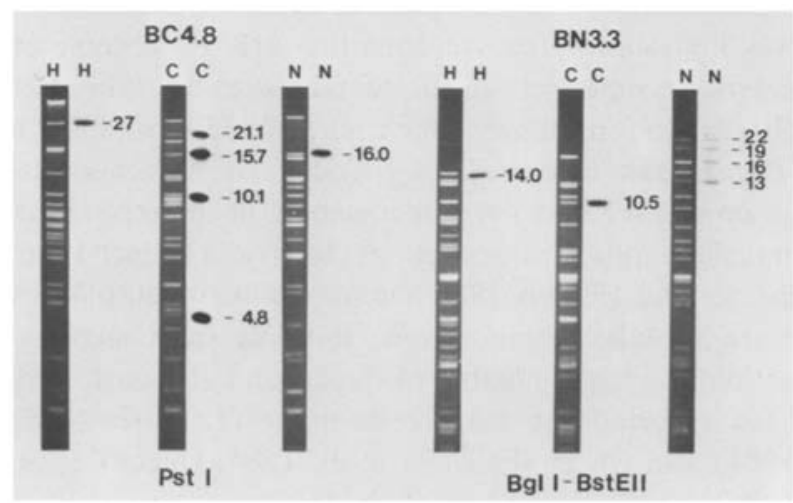

Fig. 2. Absence of recombination repeats in the $B$. hirta mitochondrial genome. BC4.8, a clone containing a $4.8 \mathrm{~kb}$ EcoRI fragment which includes the entire $B$. campestris $2 \mathrm{~kb}$ recombination repeat, and $\mathrm{BN} 3.3$, a clone containing a $3.3 \mathrm{~kb}$ PstI fragment which is internal to the $B$. nigra $7 \mathrm{~kb}$ recombination repeat, were hybridized to Zetabind filters containing PstI and Bgll-BstEII fragments of $B$. hirta $(\mathrm{H}), B$. campestris $(\mathrm{C})$, and $B$. nigra $(\mathrm{N}) \mathrm{mtDNAs}$ that had been separated on a $0.7 \%$ agarose gel

sica campestris, cv. Torch), and black mustard (Brassica nigra, USDA plant introduction line 179,860 ) by the DNAase I procedure of Kolodner and Tewari (1972). Two sources of B. hirta seed were used: a USDA plant introduction line $(195,922$; single plant selection) and seed (Durkee) purchased from the spice section of a local grocery store. MtDNA restriction profiles were idntical for the two white mustard lines with each of the five restriction enzymes used for mapping.

Uncloned $B$. hirta mtDNA restriction fragments used as hybridization probes were isolated from preparative agarose gels by electroelution, followed by phenol extraction, ether extraction, and ethanol precipitation of the isolated DNA. Cloned B. campestris mtDNA fragments used as probes are those previously described (Palmer and Shields 1984). These recombinant plasmid DNAs were isolated from $E$. coli strain JM83 by the alkaline extraction procedure of Birnboim and Doly (1979).

Restriction endonuclease digestions, agarose gel electrophoresis, bidirectional transfers of DNA fragments from agarose gels to Zetabind (AMF Cuono) and Genescreen (New England Nuclear) hybridization filters, labeling of recombinant plasmids by nick-translation, and filter hybridizations were performed as described (Palmer 1982, 1985a). Filters were stripped of hybridized probe prior to rehybridization by incubation in $0.5 \mathrm{M} \mathrm{NaOH}$ at $50^{\circ}$ for $1 \mathrm{~h}$. All filters were washed in $2 \times \mathrm{SSC}(0.3 \mathrm{M} \mathrm{NaCl})$ $30 \mathrm{mM}$ trisodium citrate) and $0.5 \%$ SDS at $65^{\circ}$ prior to autoradiography.

\section{Results}

Figure 1 shows the profile of bands produced by digestion of $B$, hirta mtDNA with the five restriction enzymes chosen for mapping studies. For each enzyme, all of the bands are of the same apparent stoichiometry, with the exception of a relatively few doublet and triplet bands. These bands were all shown to consist of two or three comigrating non-identical fragments by the mapping analysis described below (also see Fig. 4). More striking than the absence of repeated sequences as assayed by multimolar bands, is the absence of any submolar bands. Such bands were first seen in restriction digests of plant mtDNAs in 1977 (Quetier and Vedel 1977) and have since been observed in all studied genomes (Pring and Lonsdale 1985). With the advent of several complete physical maps of plant mtDNAs (Palmer and Shields 1984; Lonsdale et al. 1984; Stern and Palmer 1984a, 1986; Palmer and Herbon 1986), these submolar bands are now generally interpreted as containing repeated sequences that are engaged in high frequency intramolecular recombination. The net effect of the recombination is to place each recombining repeat element (in the case of a simple two-copy repeat family) in two different genomic environments, i.e. on two different fragments upon digestion with an enzyme that fails to cleave within the repeat. Therefore, a given repeat-containing fragment will be present in submolar concentration relative to any nonrepeat-containing fragment. The absence of submolar bands in the $B$. hirta digests (Fig. 1) suggested, therefore, the absence of high frequency intramolecular recombination in its mitochondrial genome.

To test this hypothesis, $B$. hirta mtDNA was probed with clones containing two well-characterized recombination repeats found in other Brassica species (Fig. 2). One clone, $\mathrm{BC} 4.8$, contains the entire $2 \mathrm{~kb}$ recombina- 


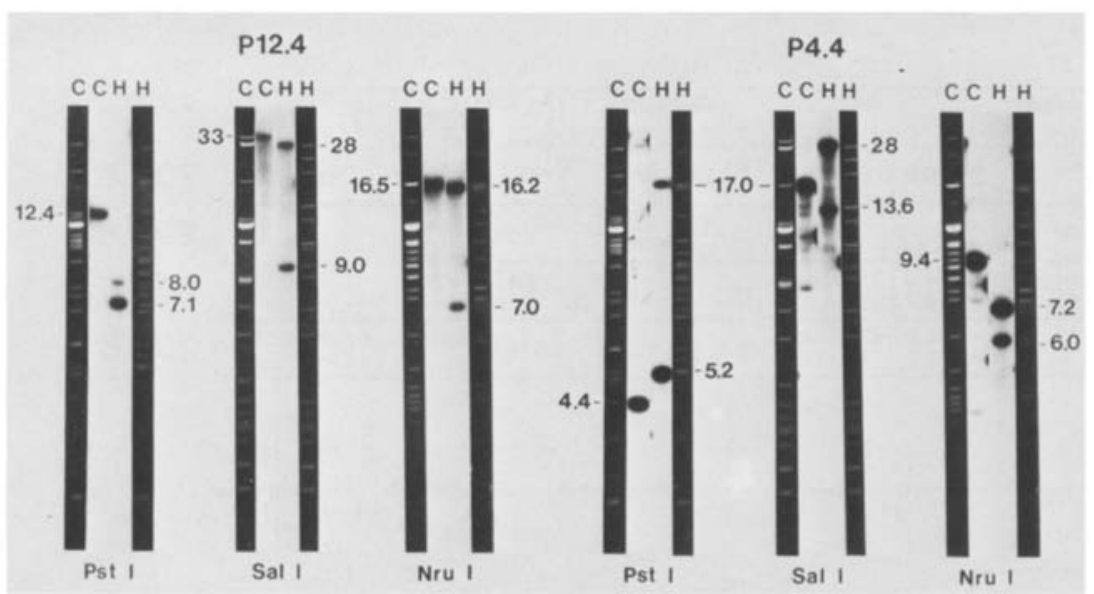

Fig. 3. Detection of mtDNA rearrangement breakpoints via hybridization between $B$. campestris and B. hirta mtDNAs. Two cloned $B$. campestris $\mathrm{mtDNA}$ restriction fragments, P12.4 and P4.4, were hybridized to Genescreen filters containing PstI, SalI and NruI fragments of $B$. campestris $(\mathrm{C})$ and $B$. hirta $(\mathrm{H}) \mathrm{mtDNAs}$ tion repeat found in B. campestris (Palmer and Shields 1984 ) and also in B. oleracea and B. napus (J. Palmer and L. Herbon, unpublished data). The other clone, BN3.3, contains a $3.3 \mathrm{~kb}$ internal portion of a $7-10 \mathrm{~kb}$ recombination repeat found in B. nigra and Raphanus sativa (Palmer and Herbon 1986). As expected, each of the recombination repeat probes hybridizes to four bands in the appropriate self-hybridization to either $B$. campestris or $B$. nigra mtDNA (Fig. 2). Furthermore, several of these bands are discernably submolar in the stained gel lanes. However, both repeat probes hybridize to only a single band in each of the $B$. hirta mtDNA lanes (Fig. 2). These results, and similar ones obtained with each of the five enzymes used in the mapping studies described below, suggest that $B$. hirta has only one copy each of the two elements that are duplicated and recombining in five other well-characterized Brassica and Raphanus mitochondrial genomes.

To determine directly whether there are any major repeated (and presumably recombining) sequences in the $B$. hirta genome, or whether the genome exists as a single size class of circles, we constructed a complete physical map of the genome by hybridization analysis. In the initial set of hybridizations, plasmids containing 96\% of the $B$. campestris mitochondrial genome were employed as heterologous probes against Genescreen filters containing $B$. hirta $\mathrm{mtDNA}$ digested with each of the five enzymes used in Fig. 1. Fifteen of the B. campestris PstI fragment clones hybridized to identicallysized PstI fragments of $B$. hirta mtDNA and provided unambiguous mapping information. However, all of the other $B$. campestris clones tested gave consistently more complex hybridization patterns with $B$. hirta mtDNA. For example, cloned $B$. campestris PstI fragments of $12.4 \mathrm{~kb}$ and $4.4 \mathrm{~kb}$ hybridize to an extra fragment in each of the B. hirta lanes shown in Fig. 3 compared to the $B$. campestris self-hybridizations. Taken together with results obtained using flanking $B$. campestris probe fragments, these findings suggest that $B$. hirta mtDNA is rearranged relative to that $B$. campestris.

A second set of mapping hybridizations was performed to resolve the ambiguities present in the heterologous mapping data. Each of the $14 \mathrm{~B}$. hirta PstI fragments (summing to $138.9 \mathrm{~kb}$ ) for which there was no comparable $B$. campestris fragment was isolated from a gel and hybridized to a filter containing various single and double digests of $B$. hirta mtDNA. These homologous hybridizations, along with the heterologous ones described above, enabled us to construct a complete physical map (Fig. 4A) incorporating all of the fragments shown in Fig. 1. The outstanding feature of the $208 \mathrm{~kb} \mathrm{~B}$. hirta mitochondrial genome is the absence of any major repeated sequences engaged in intramolecular recombination (Fig. 4A). Thus, unlike all previously characterized plant mitochondrial genomes, which are organized in a multipartite fashion, the $B$. hirta genome exists as a single size class of circular molecules.

Based on the heterologous hybridization results, we were able to align the $B$. hirta restriction map with that previously established for $B$. campestris (Fig. 4C; Palmer and Shields 1984). This alignment (Fig. 4B) shows that the two genomes differ radically in linear sequence order, confirming our earlier inference. The two genomes can be divided into no fewer than 11 regions, where sequences within each region have the same arrangment in the two genomes, but where the relative order and orientation of the regions differ between the two species (Fig. 4B). For example, one can now readily interpret the complex hybridization results shown in Fig. 3 as resulting from the dispersal to two separate regions in $B$. hirta of sequences homologous to each $B$. campestris probe fragment (Fig. 4). The extent of rearrangement in the two mtDNAs is such that we find it impossible to deduce with any confidence a single most parsimonious step-wise progression of individual rearrangements that would convert one genome into the other. Assum- 


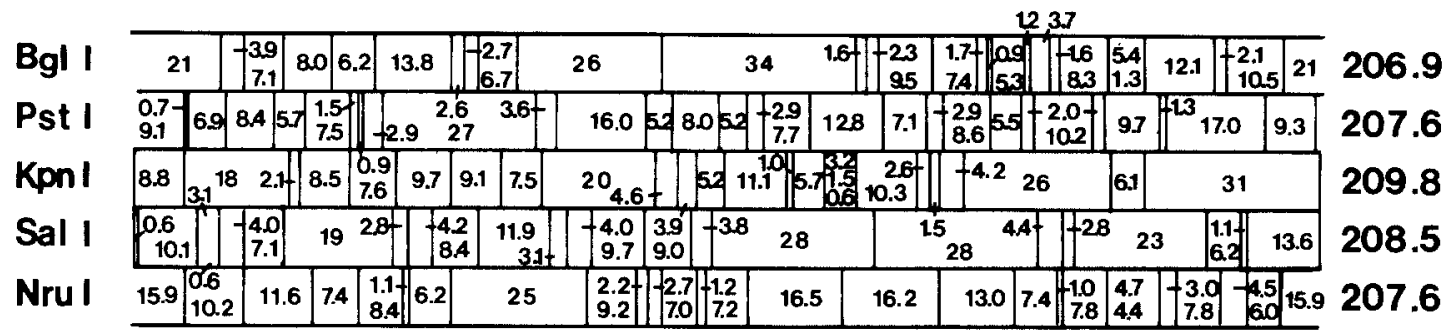

B

C

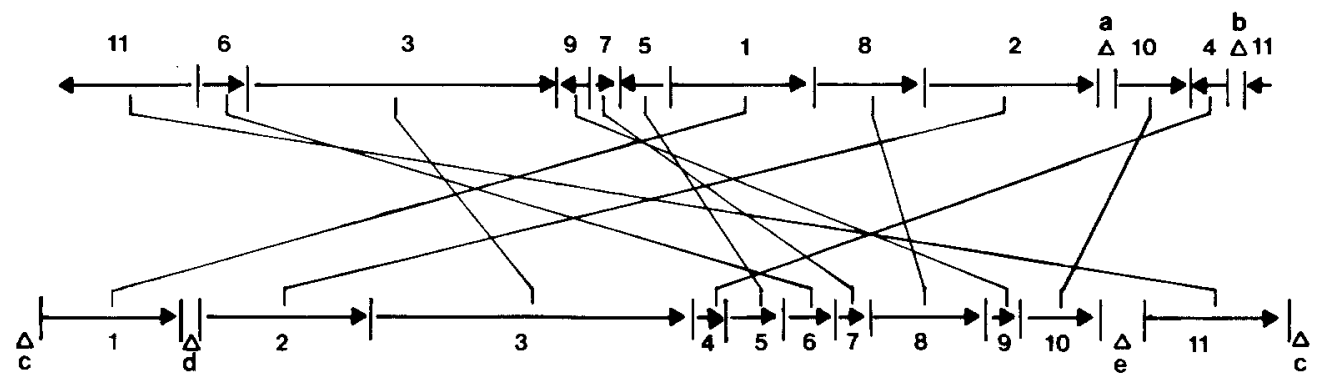

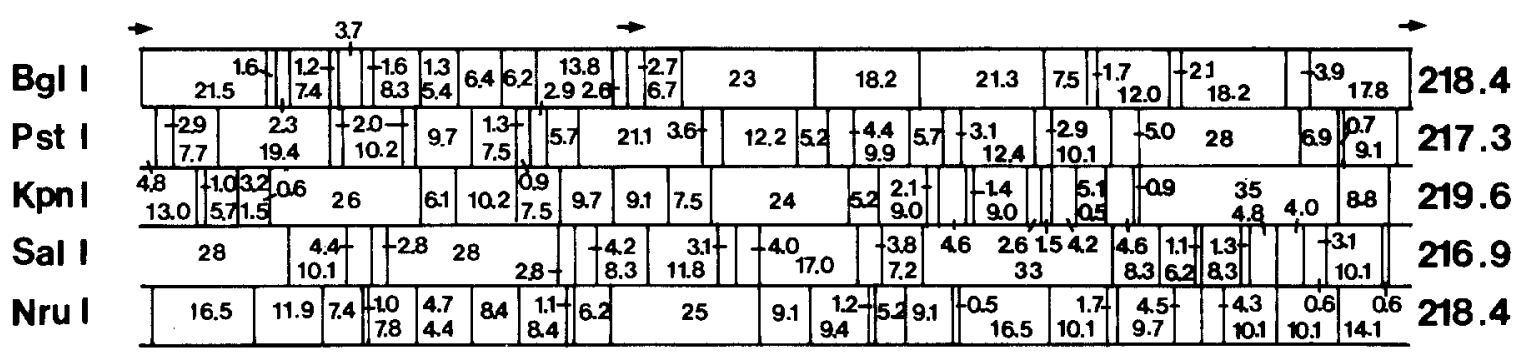

Fig. 4A-C. Restriction maps and relative arrangement of the $B$. hirta and B. campestris mitochondrial genomes. A Restriction map of the $B$. hirta mitochondrial genome. The circular map is shown linearized at SalI and KpnI sites. The numbers at the right hand end of the map indicate the summation of the restriction fragments for each of the enzymes marked at the left hand end. B Relative arrangement of homologous sequences in the B. hirta (top line) and $B$. campestris (bottom line) mitochondrial genomes. Numbers and large arrows indicate the position and relative orientation of blocks of sequences which cross-hybridize and whose arrangement has been conserved between the two genomes. The crossing lines connect homologous sequence blocks. Five major blocks of sequences which are unique to one genome or the other are indicated by the letters $a-e$ located above a " $\Delta$ ". C Restriction map of the master chromosome of $B$. campestris mtDNA (Palmer and Shields 1984). The circular map is shown linearized at a Bgll site. Arrows indicate the position and relative orientation of the two copies of the $2 \mathrm{~kb}$ repeat present on this linkage group

ing that all the rearrangements are inversions, as appears likely in comparisons involving more closely related Brassica mtDNAs (J. Palmer and L. Herbon, unpublished data), it is necessary to postulate at least 10 inversions to accomplish such a conversion.

Although the major mode of differentiation of the two genomes is rearrangement of shared sequences, they also differ by several major deletion/insertion events. Overall, the two genomes differ by $10 \mathrm{~kb}$ in size, with $B$. campestris at $218 \mathrm{~kb}$ and $B$. hirta at $208 \mathrm{~kb}$ (Fig. 4). Two $\mathrm{kb}$ of this size difference reflects the $2 \mathrm{~kb}$ sequence that is duplicated and recombining in $B$. campestris, but which is present in only one copy in $B$. hirta (Fig. 2). The remainder can be attributed primarily to four large deletions/insertions, two insertions of $3 \mathrm{~kb}$ in $B$. hirta relative to $B$. campestris $(\Delta \mathrm{a}$ and $\Delta \mathrm{b}$; Fig. $4 \mathrm{~B})$, and insertions of $3 \mathrm{~kb}$ and $8 \mathrm{~kb}$ in $B$. campestris $(\Delta \mathrm{d}$ and $\Delta \mathrm{e} ; \mathrm{Fig} .4 \mathrm{~B}$ ).
The sequences held in common by the two genomes, although highly rearranged (Fig. 4B), are virtually identical at the primary sequence level, as assayed by the proportion of shared restriction sites. We confined our analysis of restriction site conservation to the four largest regions of colinearity, regions $1-3$ and 11. B. hirta mtDNA contains 83 mapped restriction sites in these four regions, which encompass $136 \mathrm{~kb}$ of the $208 \mathrm{~kb}$ and $218 \mathrm{~kb}$ genomes (Fig. 4). Each of these 83 sites is also present in $B$. campestris mtDNA (Fig. 4; Table 1). In addition, $B$. campestris contains a unique PstI site in region 3 , resulting in fragments of $21.1 \mathrm{~kb}$ and $5.7 \mathrm{~kb}$ in B. hirta (Fig. 4). Overall, 83 of 84 restriction sites are shared between the two genomes in the $136 \mathrm{~kb}$ of DNA sequences compared (Table 1). This corresponds to an estimated $p$ value (Brown et al. 1979) of $0.20 \%$ substitutions per base pair, i.e. on the average, only 1 out of 500 base pairs differs in the two genomes. This 
Table 1. Restriction site conservation in B. hirta and B. campestris mtDNAs

\begin{tabular}{|c|c|c|c|c|c|c|c|}
\hline \multicolumn{2}{|l|}{ Region } & \multicolumn{6}{|c|}{ Sites shared/Sites compared } \\
\hline Number & Size & $\mathrm{BglI}$ & PstI & KpnI & SalI & NruI & Total \\
\hline 1 & $25 \mathrm{~kb}$ & $3 / 3$ & $3 / 3$ & $6 / 6$ & $1 / 1$ & $2 / 2$ & $15 / 15$ \\
\hline 2 & $30 \mathrm{~kb}$ & $7 / 7$ & $5 / 5$ & $2 / 2$ & $3 / 3$ & $6 / 6$ & $23 / 23$ \\
\hline 3 & $56 \mathrm{~kb}$ & $6 / 6$ & $6 / 7$ & $5 / 5$ & $7 / 7$ & $5 / 5$ & $29 / 30$ \\
\hline 11 & $25 \mathrm{~kb}$ & $2 / 2$ & $4 / 4$ & $2 / 2$ & $6 / 6$ & $2 / 2$ & $16 / 16$ \\
\hline
\end{tabular}

estimate is tentative, being based on a single restriction site difference. However, a more extensive sequence comparison gives a similar $p$ value, of $0.37 \%$ (8 mutations in 366 sites compared; J. Palmer and L. Herbon, unpublished data).

\section{Discussion}

The outstanding physical features of the $B$. hirta mitochondrial genome are the absence of high frequency intramolecular recombination, and a corresponding unicircular structure. In contrast, all other well-characterized plant mitochondrial genomes, such as those of maize (Lonsdale et al. 1984), wheat (Falconet et al. 1984; Quetier et al. 1985), and spinach (Stern and Palmer 1986), exist in a multipartite organization as the result of recombination within one or more pairs of repeats. Moreover, mtDNAs from four other Brassica species and from the closely related Raphanus sativa all contain a recombination repeat and have a tripartite structure (Palmer and Shields 1984; Palmer and Herbon 1986 and unpublished data). The singular nature of the $B$. hirta genome thus implies that, although intramolecular recombination and a multicircular organization are the common conditions for plant mtDNAs, they are by no means obligatory features of all plant mitochondrial genomes. In this sense, intramolecular recombination may be viewed as yet another dispensable and inexplicable feature of plant mtDNAs, akin to their incorporation of random bits and pieces of the chloroplast genome (Stern and Lonsdale 1982; Stern and Palmer 1984b), their large and variable sizes (Ward et al. 1981), and their frequent coexistence with a haphazard collection of small extrachromosomal plasmids (reviewed in Sederoff 1984; Palmer 1985b).

The absence of recombination repeats is just one of many structural differences between the $B$. hirta mitochondrial genome and that of other Brassica species. In particular, we find that mtDNAs of $B$. hirta and $B$. campestris, although highly similar in size and primary sequence, differ by a large number of major sequence rearrangements, on the order of 10 inversions. Extensive
mtDNA rearrangements are also found in other Brassica species (Palmer and Herbon 1986) and in Zea (Sederoff et al. 1981) and Nicotiana (Bland et al. 1985). In contrast, chloroplast DNAs from these same Brassica species are completely colinear (Palmer et al. 1983b) and most chloroplast genomes from species representing some 400 hundred million years of land plant evolution are highly similar in linear sequence order (Palmer 1985b, c; Palmer and Stein 1986; Gray 1986). It thus seems clear that the pace of structural evolution and rearrangement is much faster in plant mitochondrial genomes than in the chloroplast genome.

The $10 \mathrm{~kb}$ size difference between the $208 \mathrm{~kb} B$. hirta and the $218 \mathrm{~kb} B$. campestris genomes results largely from five major mutations - the duplication of a $2 \mathrm{~kb}$ sequence in $B$. campestris relative to $B$. hirta and four length mutations, two insertions in each genome relative to the other. If appropriate phylogenetic analysis shows that these extra sequences are indeed insertions, then they will be very interesting to examine from the standpoint of their origin. If they turn out to be derived from the chloroplast or nucleus, then, as very recently transposed sequences, they may provide important clues as to the mechanism of transposition.

The data presented in this paper indicate a very low level of primary sequence divergence between mtDNAs fo $B$. hirta and $B$. campestris. This level is several times lower than for the corresponding chloroplast DNAs (Palmer et al. 1983b), even though it is well known that chloroplast DNA has a very slow rate of nucleotide substitutions (reviewed in Curtis and Clegg 1984; Palmer 1985b). McClean and Hanson (1986) recently made the opposite conclusion regarding evolutionary rates of plant cytoplasmic genomes; they estimated that mtDNA in Lycopersicon diverges several times faster in sequence than the corresponding chloroplast DNAs (Palmer and Zamir 1982). It is possible that this apparent discrepancy may reflect actual differences in cytoplasmic evolutionary rates in the two groups of plants. However, we feel it much more likely that their mtDNA divergence values are significantly overestimated, since they are based on the "shared fragment" method of sequence comparison. A fundamental assumption of this method 
(Upholt 1977) is that divergence occurs solely by base pair substitution, yet this assumption is violated for plant mtDNAs, which undergo frequent rearrangement (see above discussion).

The comparisons presented in this paper indicate that the two plant cytoplasmic genomes have opposite tempos of evolution. Plant mtDNA evolves many times faster in structure than chloroplast DNA, yet significantly slower in primary sequence. Even more striking are the disparities in evolutionary rates between mtDNAs of plants and animals. Animal mtDNA is well known to evolve extraordinarily rapidly in primary sequence, yet is invariant in gene order among all vertebrates (reviewed in Brown 1985). These differences, as well as those involving genome size, pose quite a paradox when viewed against the similar genetic function and gene content of the mitochondrial genomes of plants and animals.

Acknowledgements. We thank J. Nugent for assistance with the mtDNA isolations, M. Hommel and the staff of the Matthaei Botanical Gardens for expert growth and care of Brassica plants, A. Srinivasan for drawing Fig. 4, and C. Makaroff and M. Zolan for ciritical reading of the manuscript. This research was funded by NIH grant GM-35087 to J. D. Palmer.

\section{References}

Birnboim HC, Doly JC (1979) Nucleic Acids Res 7:1513-1523

Bland MM, Matzinger DF, Levings CSIII (1985) Theor Appl Genet 69:535-541

Brennicke A (1980) Plant Physiol 65:1207-1210

Brown WM (1985) The mitochondrial genome of animals. In: MacIntyre RJ (ed) Monographs in evolutionary biology: Molecular evolutionary genetics. Plenum, New York, pp 95-130

Brown WM, George M Jr, Wilson AC (1979) Proc Natl Acad Sci USA 76:1967-1971

Curtis SE, Clegg MT (1984) Mol Biol Evol 1:291-301

Falconet D, Lejeune B, Quetier F, Gray MW (1984) EMBO J 3:297-302
Gray J (1986) Nature 322:501-502

Kolodner R, Tewari KK (1972) Proc Natl Acad Sci USA 69: $1830-1834$

Lebacq P, Vedel F (1981) Plant Sci Lett 20:1--9

Lonsdale DM, Hodge TP, Fauron CMR (1984) Nucleic Acids Res 12:9249-9261

McClean PE, Hanson MR (1986) Genetics 112:649-667

Palmer JD (1982) Nucleic Acids Res 10:1593-1605

Palmer JD (1985a) Meth Enzymol 118:167-186

Palmer JD (1985b) Evolution of chloroplast and mitochondrial DNA in plants and algal. In: Macintyre RJ (ed) Monographs in evolutionary biology: Molecular evolutionary genetics. Plenum, New York, pp 131-240

Palmer JD (1985c) Ann Rev Genet 19:325-354

Palmer JD, Herbon LA (1986) Nucleic Acids Res 14:9755-9765

Palmer JD, Shields CR (1984) Nature 307:437--440

Palmer JD, Stein DB (1986) Curr Genet 10:823-833

Palmer JD, Zamir D (1982) Proc Natl Acad Sci USA 79:50065010

Palmer JD, Shields CR, Cohen DB, Orton TJ (1983a) Nature $301: 725-728$

Palmer JD, Shields CR, Cohen DB, Orton TJ (1983b) Theor Appl Genet 65:181-189

Pring DR, Lonsdale DM (1985) Int Rev Cytol 97:1-46

Quetier F, Vedel F (1977) Nature 268:365-368

Quetier F, Lejeune B, Delorme S, Falconet D, Jubier MF (1985) Molecular form and function of the wheat mitochondrial genome. In: van Vloten-Doting L, Groot GSP, Hall TC (eds) Molecular form and function of the plant genome. Plenum, New York, pp 413-420

Sederoff RR (1984) Adv Genet 22:1-108

Sederoff RR, Levings CS III, Timothy DH, Hu WWL (1981) Proc Natl Acad Sci USA 78:5953-5957

Stern DB, Lonsdale DM (1982) Nature 298:698-702

Stern DB, Palmer JD (1984a) Nucleic Acids Res 12:6141-6157

Stern DB, Palmer JD (1984b) Proc Natl Acad Sci USA 81: 1946-1950

Stern DB, Palmer JD (1986) Nucleic Acids Res 14:5651-5666

Upholt WB (1977) Nucleic Acids Res 5:571-583

Ward BL, Anderson RS, Bendich AJ (1981) Cell 25:793-803

Communicated by K. P. VanWinkle-Swift

Received September 5, 1986 